\title{
Splanchnic venous thrombosis driven by a constitutively activated JAK2 V617F philadelphia-negative myeloproliferative neoplasm: a case report
}

\author{
Musa Waiswa ${ }^{1}$, Emmanuel Seremba ${ }^{1}$, Ponsiana Ocama ${ }^{1}$, Henry Ddungu ${ }^{1}$, Keneth $_{\text {Opio }}{ }^{1}$, Clement Okello $^{1}$, \\ Timothy O’shea ${ }^{2}$, Madeleine Verhovsek ${ }^{2}$, Richard Mutyabule ${ }^{3}$
}

1. Department of Medicine, Makerere University College of Health Sciences, Mulago Hospital, Kampala, Uganda

2. Department of Medicine, McMaster University, Hamilton Ontario, Canada

3. Brigham and Women's Hospital Boston.

\begin{abstract}
.
Introduction: Splanchnic venous thrombosis (SVT) has varied etiology with Philadelphia- negative myeloproliferative neoplasms (MPNs) being the most frequent underlying prothrombotic factor. Hematological indices often remain within normal range because of portal hypertension and its sequelae, causing diagnostic challenges. The high frequency of JAK2 mutation among patients with SVT reinforces the diagnostic utility of JAK2V617F testing.

Case report: We report a case of a 62-year-old black man with progressive abdominal swelling and features of decompensated chronic liver disease found to have SVT- portal vein thrombosis and how JAK2 V617F was useful in unmasking an underlying myeloproliferative neoplasm.

Conclusion: A high index of suspicion for an underlying prothrombotic factor is critical for patients presenting with thrombosis in unusual sites. This is useful in prognostic stratification and patient outcomes. JAK2 mutation screening is now part of the standard diagnostic workup in SVT.
\end{abstract}

Keywords: venous thrombosis, myeloproliferative neoplasm.

DOI: http://dx.doi.org/10.4314/ahs.v14i4.39

\section{Introduction}

Splanchnic venous thrombosis (SVT) is a rare but lifethreatening form of venous thrombosis and includes hepatic vein thrombosis (Budd-Chiari syndrome, BCS), portal vein thrombosis (PVT) and mesenteric vein thrombosis $(\mathrm{MVT})^{1}$. Involvement of two or more abdominal vein segments usually occurs ${ }^{2}$. A thrombotic event in unusual sites such as intra-abdominal veins is cause for further evaluation of an underlying hypercoagulable state, both inherited and acquired thrombophilias. A more frequent association between Philadelphia-negative myeloproliferative neoplasms (MPNs)

\section{Correspondence author: \\ Musa Waiswa \\ Department of Medicine \\ Makerere University College of \\ Health Sciences \\ P.O.Box 7072, Kampala, Uganda \\ Phone (256) 414541188 \\ Assistant clinical professor (Adjunct), \\ McMaster University, Canada \\ Email: musa600@gmail.com}

and SVT, in particular PVT and BCS, has been reported 3,4. The MPNs are a related group of diseases with a common origin of an acquired stem cell defect leading to over proliferation of the myeloid series. Diagnosis of occult forms of MPNs may be difficult in the setting of SVT because of the near normal hematological indices resulting from portal hypertension. Nearly a decade ago, it was demonstrated that MPNs are connected by an acquired somatic mutation in the JAK2 gene, the JAK2 V617F mutation ${ }^{5}$. All patients with idiopathic intra-abdominal venous thrombosis should be screened for JAK2 V617F mutation in order to detect latent or occult MPNs ${ }^{6}$.

\section{Case report}

A 62-year-old black man of Tanzanian nationality growing up in the mountainous areas of Kilimanjaro presented to Mulago hospital with a 6 weeks history of progressive abdominal swelling associated with nausea and occasional non-projectile vomiting without hematemesis. Two weeks into admission, he developed pain in the right upper quadrant of the abdomen. He affirmed complaints of poor appetite and early satiety but normal bowel movements. He reported significant loss of weight, with associated low-grade fevers but no drenching sweats. His medical history was notable for systemic 
hypertension two years prior to admission that was con- Complete blood count (CBC) on admission showed trolled on lisinopril and propranolol. He was also on a total white cell count (WBC) of 9.82 x103/uL haelow dose aspirin for the prevention of cerebrovascular moglobin of $18.5 \mathrm{~g} / \mathrm{dL}$ haematocrit of $54 \%$ and a events. He denied a history of liver disease, blood dis- rather high platelet count of $660 \times 103 / \mu \mathrm{L}$. Serological orders or cancer. He was married with four children and markers for hepatitis B virus (HBV), hepatitis C viru was an agricultural researcher. He had never smoked (HCV) and the human immunodeficiency virus (HIV) cigarettes but drank 1-2 bottles of beer on weekends. were all negative. Tumor markers including Ca-19-9,

CEA, total prostatic specific antigen (PSA) and alpha feto protein(AFP) were normal. Autoimmune screen was negative for Anti-nuclear factor and smooth muscle antibodies. S-ceruloplasmin at $33 \mathrm{mg} / \mathrm{dL}$, and prothrombin time were normal. Abdominal ultrasonography showed a splenomegaly and a hypodense lesion in the left lower lobe of the liver that revealed coagulative necrosis on histology consistent with hepatic infarction without evidence of malignancy.

Doppler ultrasound studies of the abdomen showed features of portal vein thrombosis with absent colou flow mapping in the portal veins but hepatic veins were not demonstrable. A subsequent doppler scan of both legs showed stagnation of flow in the deep calf vein and marked varicose veins bilaterally. The patient was initiated on anticoagulation with enoxaparin. A JAK2 V617F mutation assay was positive but without allele percentage. The patient while in hospital developed renal impairment, hepatic encephalopathy, and probable variceal hemorrhage leading to death.

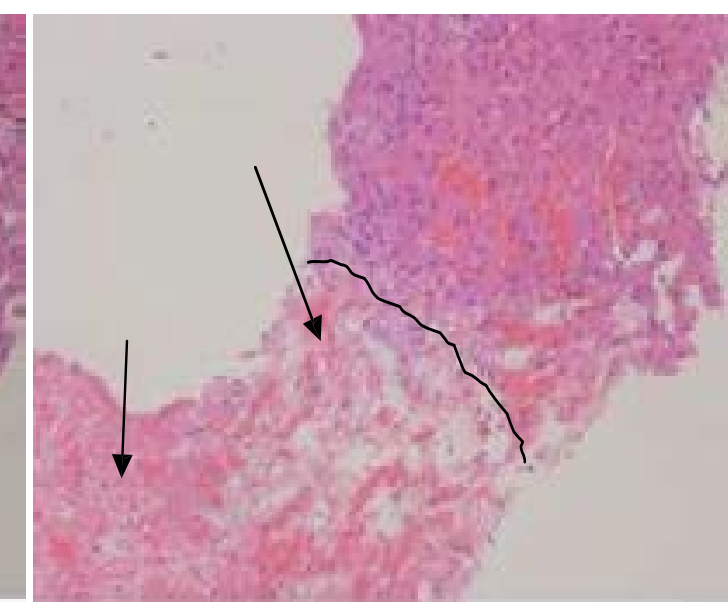
Images of liver biopsy showing sharply demarcated areas of coagulative necrosis consistent with a defined infarc.
Diagnosis transmitted electronically from Cincinnati, Ohio, USA
Discussion

Based on the new-onset SVT combined with the finding of the JAK2 V617F mutation, our patient's condition was diagnosed as an underlying chronic MPN. The clinical presentation of SVT varies according to the extent, rapidity of obstruction and venous segment involved. Specifically looking at PVT, presentation may be asymptomatic, acute or chronic. Acute thrombosis is characterized by a sudden onset of abdominal pain without evidence of chronic portal hypertension (gastrointestinal bleeding, ascites, collateral portosystemic circulation or hypersplenism). Sonography may show hyperechoic material in the vessel lumen with distension of the portal veins and its tributaries.

Doppler imaging shows the absence of flow in part or all the lumen ${ }^{7,8}$. As demonstrated in the patient, chronic PVT is a late sequela of thrombosis usually defined by the presence of a portal cavernoma with features of portal hypertension. At imaging, a diagnosis of carvernoma is readily made by abdominal imaging with ultrasound, computed tomography or magnetic resonance scan which shows serpiginous structures while the main

portal vein and or its branches are not visible ${ }^{7,9}$. Many proescale studies have been performed to study the underlying etiological factors in SVT. Both inherited and acquired thrombophilias are frequently observed in these patients. Philadelphia-negative MPNs are the most frequent underlying prothrombotic factor in BCS and PVT, with a reported prevalence of $30 \%-50 \%$ and $15 \%-30 \%$ respectively 7,10

Markers of Philadelphia-negative MPNs such as endogenous erythrocyte colony formation by bone marrow and peripheral blood cells, JAK2 mutation and the increased mRNA expression of PRV-1 in polymorphonuclear neutrophils have gained strong popularity among clinicians because it represents significant improvement in prognostic stratification and therapeutic management of patients with SVT. Other etiological factors are paroxysmal nocturnal hemoglobinuria $(\mathrm{PNH})$, autoimmune disorders, intra-abdominal inflammatory conditions, postoperative state, sickle cell anemia, anti-phospholipid syndrome, inherited hypercoagulable states (thrombophilias), including factor $\mathrm{V}$ Leiden, prohrombin G20210A mutation, protein C, protein S and anti-thrombin deficiencies.

Table 1 below shows the prevalence of thrombotic risk factors in a series of routinely investigated consecutive adult patients with non-tumorous and non-cirrhotic, acute and or

chronic PVT and BCS.

\begin{tabular}{|c|c|c|}
\hline Risk Factors & PVT patients & BCS patients \\
\hline Myeloproliferative disorders & $30 \%-40 \%$ & $40 \%-50 \%$ \\
\hline $\begin{array}{l}\text { Atypical } \\
\text { Classical }\end{array}$ & $14 \%$ & $25 \%-35 \%$ \\
\hline Antiphospholipid syndrome & $6 \%-19 \%$ & $\begin{array}{l}10 \% 0-25 \% \\
4 \%-25 \%\end{array}$ \\
\hline Paroxysmal nocturnal hemoglobinuria & $0 \%-2 \%$ & $\begin{array}{l}4 \%-5.5 \% \\
0 \%-4 \%\end{array}$ \\
\hline Behcet's disease & $0 \%-31 \%$ & $0 \%-33 \%$ \\
\hline Factor V Leiden mutation & $6 \%-32 \%$ & $6 \%-32 \%$ \\
\hline Factor II mutation & $14 \%-40 \%$ & $5 \%-7 \%$ \\
\hline Protein C deficiency* & $0 \%-26 \%$ & $10 \%-30 \%$ \\
\hline Protein S deficiency* & $2 \%-30 \%$ & $7 \%-20 \%$ \\
\hline Antithrombin deficiency* & $0 \%-26 \%$ & $0 \%-23 \%$ \\
\hline Plasminogen deficiency* & $0 \%-6 \%$ & $0 \%-4 \%$ \\
\hline Recent pregnancy & $6 \%-40 \%$ & $6 \%-12 \%$ \\
\hline Recent oral contraceptive use & & $6 \%-60 \%$ \\
\hline Hyperhomocysteinemia & $12 \%-22 \%$ & $37 \%$ \\
\hline TT677 MTHFR genotype & $11 \%-50 \%$ & $12 \%-22 \%$ \\
\hline
\end{tabular}

*Regarded as preceding the development of PVT
or BCS.

Adapted from American Association for the Study of Liver Diseases practice Guidelines, 2008

Diagnosis of MPN in the setting of SVT can however, be challenging owing to the near normal periph-

eral blood cell counts because of portal hypertension and its sequelae (splenomegaly, hemodilution and iron deficiency) ${ }^{10}$. 
The discovery of the JAK2 V617F gain-of-function mutation in 2005 , found in $95 \%$ of patients with polycythemia vera (PV) and in 50\%-60\% of patients with essential thrombocythemia and myelofibrosis, represents a crucial advance in the diagnostic approach to MPNs in the setting of SVT ${ }^{10,11}$. The goals of therapy in patients with MPNs are to avoid thrombotic and bleeding complications and also to monitor for progression to acute leukemia and myelofibrosis. In a retrospective multivariate analysis of a large cohort including patients with chronic or acute PVT, it was found that anticoagulation therapy significantly decreased the risk of recurrent thrombosis without increasing the risk of gastrointestinal bleeding ${ }^{12,13}$. Recent case series on myeloproliferative neoplasms stratify care according to thrombotic risk into low risk (defined by absence of $<6$ years), and high risk poor complane to phebotomy, or progressive myeloproliferation, i.e. splenomegaly, leucocytosis and thrombocytosis). Although our patient had no history of thrombosis, he met the high risk criteria.

In polycythemia vera, low risk patients can be managed by phlebotomy to maintain hematocrit $<0.45$ and lowdose aspirin, while high risk patients require cytoreductive therapies with hydroxyurea and interferon- $\alpha$. Busulfan can be used in elderly patients $>75$ years. For patients with essential thrombocythemia, anagrelide can be considered as second line therapy. Long term oral anticoagulation is useful in secondary prevention of thrombosis without significant increase in risk of major bleeding ${ }^{14,15}$. There is insufficient evidence in favour of interventional therapy and no consensus on the management of chronic portal vein obstruction. In case of worsening on medical therapy, patients should be considered for invasive procedures like angioplasty/ stenting, transjugular intrahepatic portosystemic shunting and liver transplantation

Hoekstra et al in their retrospective cohort study on long-term follow-up of patients with PVT and MPNs reported that mortality was primarily related to the underlying MPN and not to complications of portal hypertension ${ }^{17}$. Unfortunately our patient, though he had commenced anticoagulation and paracentesis, did not undergo phlebotomy or cytoreductive therapy. It was therefore impossible to assess the impact of these interventions on the patient's outcome.
6. Deepak A, Punamiya S, Patel N, Parekh S, Mehta S, Shah N. Prevalence of JAK29V617F) mutation in intraabdominal venous thrombosis. Tropical gastroenterology : official journal of the Digestive Diseases Foundation. 2011;32(4):279-84. Epub 2012/06/16. PubMed PMID: 22696908.

7. De Stefano V, Za T, Ciminello A, Betti S, Rossi E. Causes of adult splanchnic vein thrombosis in the mediterranean area. Mediterranean journal of hematology and infectious diseases. 2011;3(1):e2011063. Epub 2012/01/06. doi: 10.4084/mjhid.2011.063.PubMed PMID: 22220260; PubMed Central PMCID: PMCPmc3248340.

8. Van Gansbeke D, Avni EF, Delcour C, Engelholm L, Struyven J. Sonographic features of portal vein thrombosis. AJR American journal of roentgenology. 1985;144(4):749-52. Epub 1985/04/01. doi:10.2214/ aj.144.4.749. PubMed PMID: 3883708.

9. Kreft B, Strunk H, Flacke S, Wolff M, Conrad R, Gieseke J, et al. Detection of thrombosis in the portal venous system: comparison of contrast-enhanced MR angiography with intraarterial digital subtraction angiography. Radiology. 2000;216(1):86-92. Epub 2000/07/11. doi:10.1148/radiology.216.1.r00j12386. PubMed PMID: 10887231

10. Smalberg JH, Arends LR, Valla DC, Kiladjian JJ, anssen HL, Leebeek FW. Myeloproliferative neoplasms in Budd-Chiari syndrome and portal vein thrombosis: a meta-analysis. Blood. 2012;120(25):4921-8. Epub 2012/10/09. doi:10.1182/blood-2011-09-376517. PubMed PMID: 23043069

11. Campbell PJ, Green AR. The myeloproliferative disorders. The New England journal of medicine.2006:355(23):2452-66. Epub 2006/12/08 doi: 10.1056/NEJMra063728. PubMed PMID: 17151367.
2. Condat B, Pessione F, Hillaire S, Denninger MH, Guillin MC, Poliquin M, et al. Current outcome of poral vein thrombosis in adults: risk and benefit of anticoagulant therapy. Gastroenterology. 2001;120(2):490-7. Epub 2001/02/13. PubMed PMID: 11159889.

13. Riva N, Donadini MP, Dentali F, Squizzato A, Ageno

W. Clinical approach to splanchnic vein thrombosis: risk factors and treatment. Thrombosis research. 2012;130 Suppl 1:S1-3. Epub 2012/10/03. doi: 10.1016/j.thromres.2012.08.259. PubMed PMID: 23026649.

14. Barbui T, Barosi G, Birgegard G, Cervantes F, Finazzi G, Griesshammer M, et al. Philadelphia- negative classical myeloproliferative neoplasms: critical concepts and management recommendations from European LeukemiaNet. Journal of clinical oncology : official journal of the American Society of Clinical Oncology. 2011;29(6):76170. Epub 2011/01/06. doi: 10.1200/jco.2010.31.8436. PubMed PMID:21205761.

15. Barbui T, Finazzi G, Falanga A. Myeloproliferative neoplasms and thrombosis. Blood. 2013;122(13):2176-84. Epub 2013/07/05. doi:10.1182/ blood-2013-03-460154. PubMed PMID: 23823316.

16. de Franchis R. Revising consensus in portal hypertension: report of the Baveno $\mathrm{V}$ consensus workshop on methodology of diagnosis and therapy in portal hypertension. Journal of hepatology. 2010;53(4):762-8. Epub 2010/07/20. doi: 10.1016/j.jhep.2010.06.004. PubMed PMID: 20638742.

17. Hoekstra J, Bresser EL, Smalberg JH, Spaander MC, Leebeek FW, Janssen HL. Long-term follow- up of patients with portal vein thrombosis and myeloproliferative neoplasms. Journal of thrombosis and haemostasis : JTH. 2011;9(11):2208-14. Epub 2011/11/02. doi: 10.1111/j.1538-7836.2011.04484.x. PubMed PMID: 22040061.

Budd-Chiari syndrome or portal vein thrombosis. Sem-

inars in thrombosis and hemostasis. 1997;23(5):411-8.

Epub1997/01/01.doi:10.1055/s-2007-996117. PubMed PMID: 9387199

4. Valla D, Casadevall N, Lacombe C, Varet B, Goldwasser E, Franco D, et al. Primary myeloproliferative disorder and hepatic vein thrombosis. A prospective study of erythroid colony formation in vitro in 20 patients with Budd-Chiari syndrome. Annals of internal medicine.1985;103(3):329-34. Epub 1985/09/01. PubMed PMID: 4026081

5. Baxter EJ, Scott LM, Campbell PJ, East C, Fourouclas N, Swanton S, et al. Acquired mutation of the tyrosine kinase JAK2 in human myeloproliferative disorders. Lancet.2005;365(9464):1054-61.Epub2005/03/23 doi: 10.1016/s0140-6736(05)71142-9. PubMed PMID 15781101 